\title{
Application of the Sherman-Morisson formula to scattering problems by multi-component systems
}

\author{
A. Ziya Akcasu ${ }^{1}$, G. Jannink ${ }^{2}$, and H. Benoît ${ }^{3, a}$ \\ 1 Department of Nuclear Engineering and Radiological Sciences, University of Michigan, Ann Arbor, MI, 48109, USA \\ ${ }^{2}$ Laboratoire Leon Brillouin, C.E. Sacley, 91191 Gif-sur-Yvette, Cedex, France \\ 3 Institut Charles Sadron, 6 rue Boussingault, 67083 Strasbourg, Cedex, France
}

Received 5 April 2002

\begin{abstract}
The scattering matrix for multi-component systems is recalculated using the extended form of the Sherman-Morisson formula. The matrix elements are given explicitly in closed form. The Gibbs-Duhem relation separates the density and composition contributions.
\end{abstract}

PACS. 78.35.+c Brillouin and Rayleigh scattering; other light scattering - 78.40.Me Organic compounds and polymers - 02.10.Yn Matrix theory

\section{Introduction}

The classical method for the evaluation of the scattering at zero angle by multi-component systems starts with the following formulation of the scattered intensity:

$$
I=\sum_{i, j=1 . . p} b_{i} b_{j} S_{i j}
$$

where the $b_{j}$ 's are the scattering length of the $p$ species and the $S_{i j}$ are the elements of the scattering matrix $\mathbf{S}$. It has been shown [1] that $\mathbf{S}^{-1}$ can be decomposed into two terms: a density and a composition fluctuation,

$$
\mathbf{S}^{-1}=\left[\mathbf{A}+\Gamma^{-1} \mathbf{v v}^{\mathrm{T}}\right]
$$

where $\mathbf{A}$ is the matrix of the chemical potential gradients $\left(\partial \mu_{i} / \partial n_{j}\right)_{p, T, n_{k} \neq i, j}, \Gamma=V \chi_{T}, V$ being the total volume of the system, $\chi_{T}$ the isothermal compressibility, $n_{j}$ the number of molecules in the $i$-th component, and $\mathbf{v}$ the column vector of the partial volumes of the components and $\mathbf{v}^{\mathrm{T}}$ its transpose.

The problem is to invert $\mathbf{S}^{-1}$. Recently, Benoît and Jannink [1] proposed a new method for this inversion. In this paper we present a more general derivation based on the Sherman-Morisson [2] formula (SMF), i.e., which, assuming $\mathbf{A}$ to be a non-singular matrix, reads as

$$
\mathbf{S}=\mathbf{A}^{-1}-\frac{\mathbf{A}^{-1} \mathbf{v} \mathbf{v}^{\mathrm{T}} \mathbf{A}^{-1}}{\Gamma+\mathbf{v}^{\mathrm{T}} \mathbf{A}^{-1} \mathbf{v}}
$$

This identity can be verified by direct matrix multiplication. This formula was used, generically, in the

\footnotetext{
a e-mail: benoit@ics.u-strasbg.fr
}

field of multi-component polymer dynamics in the early nineties to obtain an expression for the mobility matrix in the mean-field approximation $[3,4]$. In these applications, the matrix $\mathbf{A}$ was not singular, and the application of the SMF was straightforward. In the present application, the matrix A of equation (1) is singular due to the Gibbs-Duhem rule and hence the SMF rule cannot be used directly. The purpose of this communication is thus to extend SMF to allow A to be singular, so that the expression of the static structure factor in the thermodynamic limit can be calculated in closed form even when the GibbsDuhem rule is implemented. The first part of this paper is therefore devoted to the extension of the SMF, and the second part to its application to the scattering problem.

\section{The derivation of the extended version of the SMF}

We first calculate the determinant of the matrix $\mathbf{A}+$ $\Gamma^{-1} \mathbf{v v}^{\mathrm{T}}$ (see App. A) as

$$
\left|\mathbf{S}^{-1}\right|=\left|\mathbf{A}+\Gamma^{-1} \mathbf{v} \mathbf{v}^{\mathrm{T}}\right|=|\mathbf{A}|+\Gamma^{-1} \mathbf{v}^{\mathrm{T}} \mathbf{A}^{\mathrm{ad}} \mathbf{v},
$$

where $\mathbf{A}^{\text {ad }}$ is the adjoint of $\mathbf{A}$, i.e., the transpose of the cofactor matrix of $\mathbf{A}$, and related to the inverse of $\mathbf{A}$ as

$$
\mathbf{A}^{-1}=\frac{\mathbf{A}^{\mathrm{ad}}}{|\mathbf{A}|}
$$

The elements of $\mathbf{A}^{\text {ad }}$ are expressed as

$$
A_{i j}^{\mathrm{ad}}=\frac{\partial|\mathbf{A}|}{\partial a_{j i}} .
$$


The second term in equation (3) can be expressed explicitly in terms of the elements $a_{i j}$ of $\mathbf{A}$ as

$$
\mathbf{v}^{\mathrm{T}} \mathbf{A}^{\mathrm{ad}} \mathbf{v}=\sum_{\mu=1}^{p}\left|\mathbf{C}_{\mu}\right|
$$

where

$$
\mathbf{C}_{\mu}=\left[\begin{array}{llll}
a_{11} . . & v_{1} v_{\mu} . . & a_{1 p} \\
: & : & : \\
a_{s 1} . . & v_{s} v_{\mu} . & a_{s p} \\
: & : & : \\
a_{p 1} . . & v_{p} v_{\mu} . & a_{p p}
\end{array}\right]
$$

$v_{s}$ being the partial volumes of the components at constant pressure. The matrix $\mathbf{C}_{\mu}$ is obtained by replacing elements $a_{s \mu}$ in the $\mu$-th column of $\mathbf{A}$ by $v_{s} v_{\mu}$ for $s=1 \ldots p$ as explained in Appendix A.

We then express the right-hand side of equation (1) in terms of the adjoint of $\left[\mathbf{A}+\Gamma^{-1} \mathbf{v} \mathbf{v}^{\mathrm{T}}\right]$ and its determinant using equation (4a):

$$
\mathbf{S}=\frac{1}{\left|\mathbf{A}+\Gamma^{-1} \mathbf{v} \mathbf{v}^{\mathrm{T}}\right|}\left[\mathbf{A}+\Gamma^{-1} \mathbf{v v}^{\mathrm{T}}\right]^{\mathrm{ad}} .
$$

Clearly, the front factor is just the determinant of $\mathbf{S}$ according to equation (3):

$$
|\mathbf{S}|=\frac{1}{|\mathbf{A}|+\Gamma^{-1} \mathbf{v}^{\mathrm{T}} \mathbf{A}^{\mathrm{ad}} \mathbf{v}} .
$$

The elements of the second factor in equation (6a) can be expressed as the partial derivatives of the determinant of $\left|\mathbf{A}+\Gamma^{-1} \mathbf{v v}^{\mathrm{T}}\right|$ with respect to its elements $x_{j i}=a_{j i}+\Gamma^{-1} v_{j} v_{i}$ according to equation (4b). Since the second term in $x_{j i}$ is independent of $a_{j i}$, the differentiation can be performed with respect to $a_{j i}$ only. Hence, we obtain

$$
S_{i j}=|\mathbf{S}| \frac{\partial}{\partial a_{j i}}\left(|\mathbf{A}|+\Gamma^{-1} \mathbf{v}^{\mathrm{T}} \mathbf{A}^{\mathrm{ad}} \mathbf{v}\right) .
$$

This is the desired extension of the SMF which is valid even when the matrix $\mathbf{A}$ is singular. The first term in equation (7a) is just the adjoint matrix $\mathbf{A}^{\text {ad }}$ according to equation (4b). Hence, $\mathbf{S}$ can be written as

$$
\mathbf{S}=|\mathbf{S}|\left(\mathbf{A}^{\mathrm{ad}}+\Gamma^{-1} \mathbf{Z}\right)
$$

where we have introduced the matrix $\mathbf{Z}$ to denote

$$
Z_{i j}=\frac{\partial}{\partial a_{j i}}\left(\mathbf{v}^{\mathrm{T}} \mathbf{A}^{\mathrm{ad}} \mathbf{v}\right)
$$

We mention in passing that the right-hand side of equation (7a) can be written more compactly, and interestingly, by using equation (6b) as

$$
S_{i j}=|\mathbf{S}| \frac{\partial}{\partial a_{j i}} \frac{1}{|\mathbf{S}|} .
$$

In order to express the elements $Z_{i j}$ in terms of the elements of $\mathbf{A}$ and $\mathbf{v}$, we substitute $\mathbf{v}^{\mathrm{T}} \mathbf{A}^{\text {ad }} \mathbf{v}$ from equations (5) into (8), and obtain

$$
Z_{i j}=\sum_{\mu=1}^{p} \frac{\partial\left|\mathbf{C}_{\mu}\right|}{\partial a_{j i}}
$$

We present the expression of $Z_{i j}$ explicitly for $p=2$ and 3 to illustrate the implementation of this formula.

For $p$ (number of constituents) $=2$,

$$
Z_{i j}=\frac{\partial}{\partial a_{j i}}\left|\begin{array}{cc}
v_{1}^{2} & a_{12} \\
v_{1} v_{2} & a_{22}
\end{array}\right|+\frac{\partial}{\partial a_{j i}}\left|\begin{array}{cc}
a_{11} & v_{1} v_{2} \\
a_{21} & v_{2}^{2}
\end{array}\right| \quad(i, j=1,2)
$$

or

$$
\mathbf{Z}=\left[\begin{array}{cc}
v_{2}^{2} & -v_{1} v_{2} \\
-v_{1} v_{2} & v_{1}^{2}
\end{array}\right]
$$

For $p=3$,

$$
\begin{aligned}
Z_{i j}= & \frac{\partial}{\partial a_{j i}}\left|\begin{array}{ccc}
v_{1}^{2} & a_{12} & a_{13} \\
v_{2} v_{1} & a_{22} & a_{23} \\
v_{3} v_{1} & a_{32} & a_{33}
\end{array}\right| \\
& +\frac{\partial}{\partial a_{j i}}\left|\begin{array}{ccc}
a_{11} & v_{1} v_{2} & a_{13} \\
a_{21} & v_{2}^{2} & a_{23} \\
a_{31} & v_{3} v_{2} & a_{33}
\end{array}\right|+\frac{\partial}{\partial a_{j i}}\left|\begin{array}{lll}
a_{11} & a_{12} & v_{1} v_{3} \\
a_{21} & a_{22} & v_{2} v_{3} \\
a_{31} & a_{32} & v_{3}^{2}
\end{array}\right|
\end{aligned}
$$

or

$$
\begin{aligned}
& Z_{11}=a_{33} v_{2}^{2}+a_{22} v_{3}^{2}-\left(a_{23}+a_{32}\right) v_{2} v_{3}, \\
& Z_{22}=a_{11} v_{3}^{2}+a_{33} v_{1}^{2}-\left(a_{13}+a_{31}\right) v_{1} v_{3}, \\
& Z_{33}=a_{11} v_{2}^{2}+a_{22} v_{1}^{2}-\left(a_{12}+a_{21}\right) v_{1} v_{2}, \\
& Z_{12}=a_{13} v_{2} v_{3}-a_{12} v_{3}^{2}-a_{33} v_{1} v_{2}+a_{32} v_{1} v_{3}, \\
& Z_{21}=a_{31} v_{2} v_{3}-a_{21} v_{3}^{2}-a_{33} v_{1} v_{2}+a_{23} v_{1} v_{3}, \\
& Z_{13}=a_{12} v_{2} v_{3}-a_{13} v_{2}^{2}-a_{22} v_{1} v_{3}+a_{23} v_{1} v_{2}, \\
& Z_{31}=a_{21} v_{2} v_{3}-a_{31} v_{2}^{2}-a_{22} v_{1} v_{3}+a_{32} v_{1} v_{2}, \\
& Z_{23}=a_{13} v_{1} v_{2}+a_{21} v_{1} v_{3}-a_{11} v_{2} v_{3}+a_{23} v_{1}^{2}, \\
& Z_{32}=a_{31} v_{1} v_{2}+a_{12} v_{1} v_{3}-a_{11} v_{2} v_{3}+a_{32} v_{1}^{2} .
\end{aligned}
$$

We note that the matrix $\mathbf{A}$ is not assumed to be symmetric in the derivation of the above results. When $\mathbf{A}=\mathbf{A}^{\mathrm{T}}$, we also have $\mathbf{Z}=\mathbf{Z}^{\mathrm{T}}$ so that the calculation of $Z_{i j}$ is simplified.

In conclusion, equation (9) provides a simple algorithm to calculate the elements of $\mathbf{Z}$ for any number of components.

\section{Application to scattering by multi-component systems}

\subsection{The thermodynamic modelling}

The scattering by multi-component systems has aroused great interest $[1,5-7]$, because of the information that it contains, and because of a certain degree of freedom in 
the interpretation. The modelling is based on thermodynamics: There are $p$ species, characterised by abundance's $n_{i}$, partial volumes $v_{i}$, chemical potentials $\mu_{i}, i=1, \ldots, p$. The scattering matrix $\mathbf{S}$, of order $p$, is defined by its elements

$$
S_{i j}=\left\langle\delta n_{i} \delta n_{j}\right\rangle=\delta \mu_{i} /\left.\delta n_{j}\right|_{V, T} .
$$

The derivatives are taken at constant volume and temperature. Thermodynamics allows to obtain a decomposition for the inverse scattering matrix, as in equation (1):

$$
\mathbf{S}^{-1}=\mathbf{A}+\mathbf{P}_{v}
$$

where the elements $a_{i j}$ of $\mathbf{A}$ are the increments $\delta \mu_{i} /\left.\delta n_{j}\right|_{P, T}$ taken at constant pressure and temperature. The symbol $\mathbf{P}_{v}$ means the projector on vector $\mathbf{v}$ :

$$
\left.\mathbf{P}_{v}\right|_{i j}=v_{i} v_{j} / \chi_{T} V \text {. }
$$

The Gibbs-Duhem rule writes

$$
\mathbf{A n}=0,
$$

where $\mathbf{n}=$ column $\left(n_{1}, n_{2}, \ldots, n_{p}\right)$. It has been shown $[1,6]$ that the inverse of (13), i.e., the scattering matrix, takes the form

$$
\mathbf{S}=\mathbf{P}_{n}+\mathbf{B}
$$

where $\mathbf{P}_{n}$ is the projector on vector $\mathbf{n}$,

$$
\left.\mathbf{P}_{n}\right|_{i j}=n_{i} n_{j} \chi_{T} / V
$$

and $\mathbf{B}$ is the composition fluctuation contribution. It has the property

$$
\mathbf{B v}=0,
$$

a relation conjugated to the Gibbs-Duhem rule. The problem is to determine the elements of B. Several solutions $[1,7]$ have been proposed which are complementary. Here we propose a new approach based on the extendedversion Sherman-Morisson formula derived in Section 2. This approach has the advantage to give explicit results, and it helps to gain some new insight into the problem.

\subsection{The Sherman-Morisson inversion: A re-normalized formulation}

The problem which arises in modelling the scattering experiment is to obtain equation (16) from equation (13). The extended SMF in equation (7b) gives $\mathbf{S}=|\mathbf{S}|\left(\mathbf{A}^{\text {ad }}+\Gamma^{-1} \mathbf{Z}\right)$ which we reproduce here as

$$
\mathbf{S}(\alpha)=\mathbf{S}_{0}(\alpha)+\mathbf{S}_{1}(\alpha),
$$

where

$$
\begin{aligned}
& \mathbf{S}_{0}(\alpha)=\frac{\Gamma \mathbf{A}^{\mathrm{ad}}}{\Gamma \alpha+\mathbf{v}^{\mathrm{T}} \mathbf{A}^{\mathrm{ad}} \mathbf{v}}, \\
& \mathbf{S}_{1}(\alpha)=\frac{\mathbf{Z}}{\Gamma \alpha+\mathbf{v}^{\mathrm{T}} \mathbf{A}^{\mathrm{ad}} \mathbf{v}}
\end{aligned}
$$

where $\alpha=|\mathbf{A}|$. In these equation the square matrix $\mathbf{A}$ is completely arbitrary in the sense that the elements $a_{i j}$ are all independent variables. When the Gibbs-Duhem $(\mathrm{G}-\mathrm{D})$ rule is invoked, the determinant $\alpha=0$, and equations (20) reduce to

$$
\begin{aligned}
& \mathbf{S}_{0}(0)=\frac{\Gamma \mathbf{A}^{\mathrm{ad}}}{\mathbf{v}^{\mathrm{T}} \mathbf{A}^{\mathrm{ad}} \mathbf{v}}, \\
& \mathbf{S}_{1}(0)=\frac{\mathbf{Z}}{\mathbf{v}^{\mathrm{T}} \mathbf{A}^{\mathrm{ad}} \mathbf{v}} .
\end{aligned}
$$

In order to reproduce the thermodynamic results based on the G-D rule as reported in reference [1], we have to make full use of the consequences of the G-D constraints:

$$
a_{i 1} n_{1}+a_{i 2} n_{2}+\ldots+a_{i p} n_{p}=0 \quad(i=1,2, \ldots, p),
$$

as well as the fact that in scattering problems the matrix A is symmetric. It is shown in Appendix B that, when it is done, the adjoint matrix $\mathbf{A}^{\text {ad }}$ acquires the following delightfully simple form:

$$
\mathbf{A}^{\mathrm{ad}}=\frac{A_{11}^{\mathrm{ad}}}{n_{1}^{2}} \mathbf{n n}^{\mathrm{T}}
$$

where $\mathbf{n}$ is the column vector $\left(n_{1}, n_{2}, \ldots, n_{p}\right)$. This result was obtained in reference [1] in the case of $p=3$. Substituting equation (24) into equation (21), and using $V=\mathbf{n}^{\mathrm{T}} \mathbf{v}$, where $V$ is the volume of the system, we obtain

$$
\mathbf{S}_{0}(0)=\frac{\Gamma}{V^{2}} \mathbf{n n}^{\mathrm{T}}
$$

which is identical to the projector $\mathbf{P}_{n}$ defined in equation (17), with $\Gamma=V \chi_{T}$.

The other contribution $\mathbf{B}$ in equation (16), which is related to the composition fluctuations, is identical to $\mathbf{S}_{1}(0)$ in equation (22). Hence, using $\mathbf{v}^{\mathrm{T}} \mathbf{A}^{\mathrm{ad}} \mathbf{v}=\left(V^{2} / n_{1}^{2}\right) A_{11}^{\mathrm{ad}}$, we get

$$
B_{i j}=\frac{n_{1}^{2}}{V^{2} A_{11}^{\mathrm{ad}}} Z_{i j},
$$

where $Z_{i j}$ are given by equation (9) in general. The use of G-D constraints in the calculation of $B_{i j}$ does not lead to any simplification. We present explicit results only for $p=2$ and $p=3$ as an illustration. For $p=2, A_{11}^{\text {ad }}=a_{22}$, and $Z_{i j}$ are given in equation (10):

$$
\mathbf{B}=\frac{n_{1}^{2}}{V^{2} a_{22}}\left[\begin{array}{cc}
v_{2}^{2} & -v_{1} v_{2} \\
-v_{1} v_{2} & v_{1}^{2}
\end{array}\right]
$$

which is a standard result.

For $p=3, A_{11}^{\text {ad }}=a_{22} a_{33}-a_{12}^{2}$, and $Z_{i j}$ are given in equation (11). We present only $B_{11}$ as an example:

$$
B_{11}=\frac{n_{1}^{2}}{V^{2}\left(a_{22} a_{33}-a_{23}^{2}\right)}\left(a_{33} v_{2}^{2}+a_{22} v_{3}^{2}-2 a_{23} v_{2} v_{3}\right),
$$

which was also obtained in reference [1].

We note that the property in equation (18), i.e., $\mathbf{B v}=0$, is easily verified in the case of $p=2$. The proof 
$\mathbf{B v}=0$, or, equivalently, $\mathbf{Z v}=0$, for an arbitrary number of components is based on the following identity:

$$
\frac{\partial^{2}|\mathbf{A}|}{\partial a_{j i} a_{\nu \mu}}=-\frac{\partial^{2}|\mathbf{A}|}{\partial a_{\nu i} a_{j \mu}},
$$

which follows from the fact that the simultaneous interchange of the indices $j$ and $\nu$, and the interchange of the $j$-th and $\nu$-th rows of $|\mathbf{A}|$ implies differentiation with respect to the same elements. The minus sign comes from the interchange of two rows in a determinant. The following steps are now self-explanatory:

$$
\begin{aligned}
Z_{i j} v_{j}= & v_{j} v_{\nu} v_{\mu} \frac{\partial^{2}|\mathbf{A}|}{\partial a_{j i} a_{\nu \mu}}=v_{j} v_{\mu} v_{\nu} \frac{\partial^{2}|\mathbf{A}|}{\partial a_{\nu i} a_{j \mu}}= \\
& -v_{j} v_{\mu} v_{\nu} \frac{\partial^{2}|\mathbf{A}|}{\partial a_{j i} a_{\nu \mu}}=-Z_{i j} v_{j} .
\end{aligned}
$$

A general method of computation of the scattering by multi-component, compressible systems has been presented. It assumes that the chemical potential gradients at constant pressure are known.

\subsection{Discussions}

The decomposition of the scattering matrix $\mathbf{S}$, re-derived with a new method in the preceding sections, calls for the following comments:

1) This decomposition is given for any value of $\alpha=$ Det $\mathbf{A}$. The case $\alpha=0$ corresponds to constant pressure and temperature conditions. The case $\alpha \neq 0$ is not yet fully interpreted but could correspond to an adiabatic situation.

The condition $\alpha=0$ is a necessary condition for the rule to be obeyed: It produces a separation into a density fluctuation matrix and a composition fluctuation matrix. On the contrary, when $\alpha \neq 0$, the rule is not obeyed, and it is not possible to partition $\mathbf{S}$ into density and composition contributions.

2) The fact that the scattering matrix $\mathbf{S}$ may be decomposed into a density and a composition fluctuation sub-matrices does not automatically imply that density and composition fluctuations are uncorrelated. For this to occur it is necessary that $\mathbf{S}$ be represented as a direct sum [8]:

$$
\mathbf{S}=\mathbf{B} \oplus \mathbf{P}_{n}
$$

Such a situation exists, when, for instance, $\mathbf{B}$ is the inverse of the restriction of $\mathbf{A}$ to the subspace orthogonal to vector " $n$ " [7]. The matrix $\mathbf{P}_{n}$ being the projector on "n", there is no intersection between the respective subspaces. This representation allows uncorrelated fluctuations [9], which could perhaps be observed experimentally.

3) The property $\mathbf{B v}=0$ is a key relation for the introduction of contrasts. It is however not the only one, if one considers the fact that the scattered intensity is a quadratic form of the scattering matrices: One can imagine many transformations which would leave the intensity unchanged.

\section{Appendix A. Calculation of the determinant of $S^{-1}$}

We start with the expansion of $\left|\mathbf{S}^{-1}\right|$ :

$$
\begin{aligned}
\left|\mathbf{S}^{-1}\right|= & \sum_{P}(-1)^{P}\left(a_{1 k_{1}}+\Gamma^{-1} v_{1} v_{k_{1}}\right) \\
& \times\left(a_{2 k_{2}}+\Gamma^{-1} v_{2} v_{k_{2}}\right) \ldots\left(a_{p k_{p}}+\Gamma^{-1} v_{p} v_{k_{p}}\right),
\end{aligned}
$$

where the symbols have their usual meanings. Upon expansion we get

$$
\begin{aligned}
\left|\mathbf{S}^{-1}\right|= & |\mathbf{A}|+\frac{1}{\Gamma} v_{1} \sum_{P}(-1)^{P} v_{k_{1}} a_{2 k_{2}} \ldots a_{p, k_{p}}+\ldots \\
& +\frac{1}{\Gamma} v_{p} \sum_{P}(-1)^{P} a_{1 k_{1}} \ldots a_{p-1, k_{p-1}} v_{k_{p}} \\
& +\frac{1}{\Gamma^{2}}\left\{v_{1} v_{2} \sum_{p}(-1)^{P} v_{k_{1}} v_{k_{2}} a_{3 k_{3}} \ldots a_{p k_{p}}+\ldots\right\} .
\end{aligned}
$$

The terms involving $(1 / \Gamma)^{2}$ and the higher powers are zero because they involve determinants with two identical rows. The sum of the terms involving $1 / \Gamma$ is equal to $(1 / \Gamma) \mathbf{v}^{\mathrm{T}} \mathbf{A}^{\mathrm{ad}} \mathbf{v}$. Hence equation (3) follows.

To calculate $\mathbf{v}^{\mathrm{T}} \mathbf{A}^{\text {ad }} \mathbf{v}$ in terms of the elements $a_{i j}$ and $v_{s}$, we start with the matrix identity $a_{\nu \mu} A_{\mu \nu}^{\text {ad }}=|\mathbf{A}|$ for any given $\mu$ with summation on $\nu$. This is the expansion of the determinant $|\mathbf{A}|$ into the elements of the $\mu$-th column. Thus, $v_{\nu} A_{\mu \nu}^{\text {ad }}=\left|\mathbf{X}_{\mu}\right|$ is the determinant of a matrix $\mathbf{X}_{\mu}$ which is obtained by replacing the elements $a_{\nu \mu}$ in the $\mu$-th column of $|\mathbf{A}|$ by $v_{\nu}, \nu=1,2, \ldots p$. Consider now the summation $v_{\mu}\left|\mathbf{X}_{\mu}\right|$ for $\mu=1,2 \ldots p$. Each term in this summation, say the $\mu$-th term, is the determinant of a matrix $\mathbf{C}_{\mu}$, which is obtained by replacing the elements $a_{\nu \mu}$ in the $\mu$-th column of $|\mathbf{A}|$ by $v_{\mu} v_{\nu}, \nu=1,2, \ldots p$. Hence, $\mathbf{v}^{\mathrm{T}} \mathbf{A}^{\mathrm{ad}} \mathbf{v}=v_{\mu} A_{\mu \nu}^{\mathrm{ad}} v_{\nu}=\sum_{s=1}^{p}\left|\mathbf{C}_{s}\right|$ has been established, proving equations (5) in the text.

\section{Appendix B. Implementation of the Gibbs-Duhem relation in the SMF}

The G-D relations are expressed as $a_{i j} n_{j}=0, i=$ $1,2, \ldots, p$, where summation on $j$ is implied. For a given set of values of $n_{j}$, we can solve these equations for the diagonal terms as

$$
a_{i i}=-\frac{1}{n_{i}} \sum_{j \neq i} a_{i j} n_{j}
$$

where we treat $a_{i j}$ as $p(p-1)$ independent variables for $i \neq j$. Since $\mathbf{A n}=0$ implies $|\mathbf{A}|=0$, we have

$$
\sum_{j=1}^{p} a_{i j} A_{j i}^{\mathrm{ad}}=0, \quad i=1,2, \ldots, p
$$


where $A_{j i}^{\text {ad }}$ does not depend on $a_{i k}$ or $a_{k j}$ for any $k$ by its definition as adjoint. The diagonal term is given by

$$
a_{i i} A_{i i}^{\mathrm{ad}}=-\sum_{j=1 \neq i}^{p} a_{i j} A_{j i}^{\mathrm{ad}} .
$$

Substituting $a_{i i}$ from above, we find

$$
\sum_{j=1 \neq i}^{n} a_{i j}\left[\frac{n_{j}}{n_{i}} A_{i i}^{\mathrm{ad}}-A_{j i}^{\mathrm{ad}}\right]=0 .
$$

Since $a_{i j}$ are independent variables and the coefficients do not depend on them, we obtain

$$
\frac{A_{j i}^{\mathrm{ad}}}{n_{j}}=\frac{A_{i i}^{\mathrm{ad}}}{n_{i}}, \quad \text { for any } i \text { and } j
$$

which can also be written as $A_{j i}^{\text {ad }} / n_{j}=A_{k i}^{\text {ad }} / n_{k}$ for any $j$ and $k$ for a given $i$. Expressing the off-diagonal elements in terms of the diagonal elements using equation (B1), we can express the elements of the adjoint matrix $\mathbf{A}^{\text {ad }}$ as

$$
\left[\mathbf{A}^{\mathrm{ad}}\right]_{\mu \nu}=\frac{n_{\mu}}{n_{\nu}} A_{\nu \nu}^{\mathrm{ad}}
$$

So far, we have not assumed that the matrix $\mathbf{A}$ is symmetric. When $\mathbf{A}=\mathbf{A}^{\mathrm{T}}$, we also have $A_{j i}^{\mathrm{ad}}=A_{i j}^{\mathrm{ad}}$.
When substituted into equation (B1), the latter yields

$$
A_{j i}^{\mathrm{ad}}=\left(n_{j} / n_{i}\right) A_{i i}^{\mathrm{ad}}=A_{i j}^{\mathrm{ad}}=\left(n_{i} / n_{j}\right) A_{j j}^{\mathrm{ad}} .
$$

So, the diagonal elements satisfy $A_{i i}^{\text {ad }}=\left(n_{i} / n_{j}\right)^{2} A_{j j}^{\text {ad }}$ or $A_{i i}^{\text {ad }}=\left(n_{i} / n_{1}\right)^{2} A_{11}^{\text {ad }}$, i.e., they can be expressed only in terms of one of them. Using this result in equation (B2), we find

$$
\left[\mathbf{A}^{\mathrm{ad}}\right]_{\mu \nu}=\frac{n_{\mu} n_{\nu}}{n_{1}^{2}} A_{11}^{\mathrm{ad}}
$$

which leads to equation (24) in the text.

\section{References}

1. H.C. Benoît, G. Jannink, Eur. Phys. J. E 3, 283 (2000).

2. G.H. Golub, Van Loan C.F., Matrix Computations (The Johns Hopkins University Press, Baltimore, 1989).

3. A.Z. Akcasu, M. Tombakoglu, Macromolecules 23, 607 (1990).

4. M. Tombakoglu, A.Z. Akcasu, Polymer 33, 1127 (1992).

5. T.L. Hill, An Introduction to Statistical Thermodynamics (Addison Wesley, 1960) p. 182.

6. J.S. Higgins, H.C. Benoît, Polymers and Neutron Scattering (Oxford University Press, 1994).

7. J. des Cloizeaux, G. Jannink, Physica A 102, 120 (1980).

8. P.R. Halmos, Finite-Dimensional Vector Spaces (SpringerVerlag, New York, 1983).

9. P.D. Gujrati, J. Chem. Phys. 112, 4806 (2000). 